\title{
Optical Appearance of Dynamical Models for Circumstellar Dust Shells around Long-Period Variables: AFGL 3068
}

\author{
JAN MARTIN WINTERS ${ }^{1}$, AXEL J. FLEISCHER ${ }^{1}$, \\ THIBAUT LE BERTRE ${ }^{2}$, and ERWIN SEDLMAYR ${ }^{1}$ \\ 1 Institut für Astronomie und Astrophysik, TU Berlin, Germany \\ 2 Observatoire de Paris, DEMIRM, Paris, France
}

Based on dynamical models of circumstellar dust shells (CDS) around longperiod variables (LPVs), including time-dependent hydrodynamics and a detailed treatment of the processes of formation, growth and evaporation of carbon grains, angle- and frequency-dependent radiative transfer calculations have been carried out. The models are completely determined by 6 parameters, comprising the 4 fundamental stellar parameters $\left(M_{*}, T_{*}, L_{*}, \epsilon_{\mathrm{i}}\right)$ and the pulsation period and velocity amplitude at the base of the atmosphere $\left(P, \Delta u_{\mathrm{P}}\right)$. It turns out that the discrete onion-like structure of the CDS (see Fleischer et al., this volume) decisively influences the shape of the synthetic light curves as well as the calculated spatial intensity profiles and the corresponding spatial spectra. We present a consistent theoretical model for the CDS of the extreme carbon star AFGL 3068. The synthetic light curves, which are multiperiodic on a timescale of approximately 3 pulsation periods, the spectral energy distributions at different phases, and the terminal wind velocity are in excellent agreement with the observed properties of this object. Therefore, the values of the fundamental stellar parameters and the distance to the object, which are not directly accessible by observation, can be derived from this model: $M_{\star}=0.9 M_{\odot}, L_{\star}\left(t_{0}\right)=13000 L_{\odot}, T_{\star}\left(t_{0}\right)=2100 \mathrm{~K}$, carbonto-oxygen ratio $\epsilon_{\mathrm{C}} / \epsilon_{\mathrm{O}}=1.33$ (the other element abundances are assumed to be solar), $P=696 \mathrm{~d}$, and $\Delta u=8.0 \mathrm{~km} \mathrm{~s}^{-1}$. This model yields a final outflow velocity $v_{\infty}=15.7 \mathrm{~km} \mathrm{~s}^{-1}$, a mass-loss rate $\dot{M}=1.7 \times 10^{-4} M_{\odot} \mathrm{yr}^{-1}$, a dustto-gas ratio $\rho^{\mathrm{d}} / \rho^{\mathrm{g}}=1.6 \times 10^{-3}$, and a distance to AFGL 3068 of $d=1.3 \mathrm{kpc}$. A temporal evolution of the shell is seen in the calculated brightness profiles and in the corresponding visibility functions. Therefore, to obtain a further test of the model, it would be valuable to observe the brightness distribution of AFGL 3068 directly with very high angular resolution $(\approx 0.01 \mathrm{arcsec})$.

This poster, including its figures, may be seen at

http://export.physik.tu-berlin.de/Publikationen/

This work was supported by the BMBF (grant 05 3BT13A 6) and by the DFG (grant Se 420/8-1). 